\title{
Trends in microbial profile of burn patients following an event of dust explosion at a tertiary medical center
}

\author{
Yin-Yin Chen ${ }^{1,2,3}$, Ping-Feng Wu ${ }^{4,5}$, Chii-Shya Chen ${ }^{6}$, lan-Horng Chen ${ }^{6}$, Wan-Tsuei Huang ${ }^{1,2}$ and \\ Fu-Der Wang ${ }^{1,4,5,7^{*}}$ (iD
}

\begin{abstract}
Background: Microbial infection is the main cause of increased morbidity and mortality in burn patients, especially infections caused by multiple drug-resistant organisms (MDRO). The purpose of this study was to explore major microbial trends in burn patients.

Methods: This retrospective study was conducted at burn wards and intensive care units, where burn patients were admitted following an event of dust explosion. Data were collected for a number of variables including severity of burns, demographic and clinical characteristics, laboratory data, and therapeutic devices.

Results: A total of 1132 specimens were collected from 37 hospitalized burn patients with mean TBSA of 46.1\%.The most commonly isolated species were Staphylococcus spp. (22.4\%). The highest rate of antibiotic resistance was observed in carbapenem-resistant A. baumannii (14.6\%), followed by methicillin-resistant S. aureus (11.3\%). For each additional 10\% TBSA, the isolation of MDRO increased 2.58-17.57 times $(p<0.05)$; for each additional $10 \%$ of the third-degree burn severity, the risk of MDRO significantly decreased by $47 \%(95 \% \mathrm{Cl}, 0.38-0.73, p<0.001)$ by Cox model.

Conclusions: The proportion of overall microbial isolates increased with the increase in TBSA and duration of time after burns. The extent of TBSA was the most important factor affecting MDRO.
\end{abstract}

Keywords: Burn, Infection, Microbial

\section{Background}

Generally, microorganisms will colonize and grow quickly after burns due to the loss of the skin barrier [1]. In burn patients, potential biomarkers can be used clinically to identify infections and sepsis; they can also be used to predict the survival of injuries, monitor the severity of injuries, organ function or wound healing. There are several risk factors which facilitate microbial

\footnotetext{
* Correspondence: fdwang@vghtpe.gov.tw

'Department of Infection Control, Taipei Veterans General Hospital, Taipei, Taiwan

${ }^{4}$ Division of Infectious Diseases, Department of Medicine, Taipei Veterans General Hospital, Taipei, Taiwan

Full list of author information is available at the end of the article
}

colonization and infection, including age and comorbidities, burn wound size, impaired immunity (e.g., hyperglycemia, hypermetabolic response), and medical measures (e.g., use of invasive catheters, transfusion, delays in burn wound excision) etc. $[1,2]$. The microbial colonizers or pathogens affecting burn patients include bacteria and fungi $[3,4]$. The most common Gram-positive bacteria implicated in burn wound infections include Staphylococcus spp., Enterococcus spp., and beta-hemolytic Streptococcus group A. Among that group, Staphylococcus aureus continues to be one of the most important bacterial cause of burn wound infections [4-7]. The most frequently isolated Gram-negative bacteria from

(C) The Author(s). 2020 Open Access This article is licensed under a Creative Commons Attribution 4.0 International License, which permits use, sharing, adaptation, distribution and reproduction in any medium or format, as long as you give appropriate credit to the original author(s) and the source, provide a link to the Creative Commons licence, and indicate if changes were made. The images or other third party material in this article are included in the article's Creative Commons licence, unless indicated otherwise in a credit line to the material. If material is not included in the article's Creative Commons licence and your intended use is not permitted by statutory regulation or exceeds the permitted use, you will need to obtain permission directly from the copyright holder. To view a copy of this licence, visit http://creativecommons.org/licenses/by/4.0/ The Creative Commons Public Domain Dedication waiver (http://creativecommons.org/publicdomain/zero/1.0/) applies to the data made available in this article, unless otherwise stated in a credit line to the data. 
patients with burn wounds include Pseudomonas aeruginosa, Acinetobacter baumannii, Klebsiella spp., Stenotrophomonas spp., Escherichia coli, and Enterobacter cloacae $[1,2,5,7,8]$.

Patients with severe burns are more prone to infections caused by multiple drug-resistant organisms (MDRO); common examples include methicillin-resistant Staphylococcus aureus (MRSA), vancomycin-resistant Enterococcus (VRE), MDR Pseudomonas spp. and Acinetobacter spp. [5, 9]. The care and treatment of these patients have been quite challenging. If some of the infection control measures are neglected, these pathogens may even cause an outbreak in burn units $[3,10]$. These infections result in prolonged hospital stay and high mortality rates in patients with burns. Additionally, the cost of medical care for burn patients is substantial [11-13].

A number of previous studies used databases to conduct and publish clinical epidemiological data on populations of burn patients that mostly involved groups of children with varying degrees of burns [14-17]. Thus, the population analysis of these different databases exhibited more heterogeneity. One sudden dust (flammable starch-based powder) explosive event occurred at a recreational water park in northern Taiwan. More than 4000 young attendees went to the music party where the incident happened on stage. As a result, 499 people were injured, 393 were hospitalized and 221 of them ended up in the intensive care units (ICUs) of 46 hospitals in seven cities. As most attendees wore flammable swimwear, the patients had large total body surface area (TBSA, average $41 \%$ ) of burns. A total of 281 people suffered more than $40 \%$ injuries; of those, 41 victims were severely injured with TBSA more than $80 \%$, and 15 fatalities were attributed to the explosion [18]. Among the hospitalized, 44 patients with similar age group and exposure levels were enrolled in the study.

There have been limited published reports addressing changes in trends among various important microorganisms isolated from burn patients. We hypothesized that various important microorganisms and their MDRO would show different trends due to differences in burn severity and time. Therefore, the purpose of our study was to determine the isolation rates of common microorganisms and MDRO, and their fluctuations and trends in TBSA range as well as post-burn times using stratified trends and multiple analyses.

\section{Methods}

\section{Hospital and cases}

This retrospective observational study, using an active hospital-wide HAI surveillance from June 27, 2015 to October 31, 2015 was conducted at a burn department (5 bed-ICU and 14 bed-ward), four other ICUs of a 3045- bed tertiary referral medical center in northern Taiwan. The study protocol was approved by the Institutional Review Board of the research hospital, and the requirement for obtaining informed consent was waived.

After the event of the dust explosion on the evening of June 27, 2015, 44 injured patients were immediately sent to our emergency department for medical treatment. Eight victims were discharged after treatment for minor wounds, while 36 victims with severe burns needed to be hospitalized for treatment and care. On the following day, hospitalized burn cases were further evaluated for graded assessment of the severity of burns. Afterwards, the case beds were rearranged from the original emergency hospitalization to the dispersal of wards (16 ICUs or wards) and to centralized ward care (5 ICUs and 1 ward). Two cases were transferred from other hospitals on June 28 and 29 and one victim was transferred to another hospital on July 1 . All patients hospitalized from a period of June 27 to 29,2015 due to the dust explosion were eligible for the study. Those cases that were discharged from the hospital, but readmitted later, were excluded from the study.

\section{Strategies for infection control}

When patients were transferred to the emergency department, an interdisciplinary medical team was quickly established and held regular meetings on treatment and disposal strategies. An infectious disease physician also participated in the antimicrobial stewardship including assessments and medications. In addition, sufficient healthcare workers and appropriate administrative support and management staff was provided.

Cases underwent escharotomy or debridement to remove damaged tissue in operation room. Human skin or fish skin were provided for graft to treat severe burns. The choice of dressing type was determined by the attending physician of the interdisciplinary team depending on the depth, size, exudate, and infection of the burn wound. For example, AQUACEL dressings (ConvaTec Inc., UK) were used for large-scale third burn-degree; and AQUACEL Ag + dressings which the power to disrupt and destroy biofilm were used for infected wounds. Dressing changes for all cases, if required, were carried out at their bedsides.

The prevention and control practices for burn infections have been devised according to recommendations from published guidelines $[1,2,9]$. The healthcare personnel and visitors were required to strictly adhere to the infection control procedures. Protocols were also developed in accordance with standard procedures for environmental cleaning, disinfection procedures, and training of housekeeping staff to perform cleaning and disinfection. During this study, the infection control practitioners (ICPs) used a checklist to improve the 
auditing of the cleaning personnel's correct implementation of equipment and the environment cleaning and disinfection. An adenosine triphosphate (ATP) luminometer was also used to measure the level of cleanliness. When the ATP measurements were lower than the cleaning standard value $(<100$ relative light unit), the microbial surveillance of the indoor environment and the equipment would be carried out, and it must be cleaned again until the cleaning standard value were met. Additionally, a laboratory-based alert system was established that immediately notified ICPs and clinical personnel when new MDRO were isolated from cases.

\section{Data collection}

Data on the severity of burns and frequencies of surgery, demographic and clinical characteristics, laboratory data and therapeutic devices used were recorded. Additionally, revised Baux score, calculated as the sum of the age and TBSA was also included. Baux scores were increased by 17 and 0 for all cases with inhalation injuries and without inhalation injuries, respectively (see Tables 1 and 2). If no clinically significant microorganism was isolated, empirical antimicrobial treatment, such as a carbapenem plus glycopeptide, was administered for $<7$ days to hospitalized patients with severe sepsis or septic shock and a large burn area [19]. Antifungal agent (fluconazole or echinocandin, if fluconazole was contraindicated) was administered at 7 days after the burn, if the patient exhibited at least three specific risk factors.

The standard surveillance protocols and healthcareassociated infection (HAI) site definitions were according to the National Healthcare Safety Network of the Centers for Disease Control and Prevention [20]. Culturing of pathogens was performed when the patient had a temperature over $38^{\circ} \mathrm{C}$ or a fever of unknown etiology, exudates or pus from an insertion site or surgical site, or signs and symptoms of suspected infections. The microbial culture of burn wounds was judged by the physician for the degree of the wound dirty and the signs or symptoms of infection. Specimens of a burn wound were usually collected after the wound has been cleaned during the patient's operation. Microbiological work was carried out in a microbiology laboratory that was certified internationally through the College of American Pathologists. Identification of all microbial isolates was confirmed at the laboratory using automated-method employing identification cards of VITEK-MS system (BioMerieux Inc., Mercy L'etoil, France). The susceptibility results were interpreted based on the criteria specified by the Clinical and Laboratory Standards Institute 2015. Cultures reported with intermediate sensitivity were considered resistant.

\section{Statistical analysis}

A descriptive analysis of all collected variables was performed. For any given case if same species were isolated from the same site on multiple occasions, they were recorded once only. Microbiological profile was established including type of bacteria and antibiotic susceptibility. Stratified analyses of TBSA and days after admission were used. The Chi square test for linear trend was used to examine the trends in main microbial isolates in TBSA and days after admission. The simple logistic regression and the simple Cox regression analyses were also used. Next, the multiple logistic regression and the multiple Cox regression with an enter approach were used to assess risk factors of MDRO, while adjusting for potentially confounding variables such as TBSA, and burn-degree severity. Odds ratios (OR), hazard ratio (HR), and 95\% confidence intervals (CI) were calculated. All tests were two-tailed, and $p$ values less than 0.05 were considered statistically significant.

\section{Results \\ Patient demographic data}

A total of 37 severely hospitalized burn cases were admitted during the study period. Of these, 24 cases $(64.9 \%)$ needed to stay in the ICUs for treatment. The mean percentage of initial TBSA was $46.1 \% \pm 29.1 \%$ (median $50 \%$, range $5-92 \%$; $>50 \% 21$ cases) and 16 cases had more than $50 \%$ third-degree burns (43.2\%). The main sites with $30-50 \%$ of third-degree burns were upper limbs (except wrist and hand; 48.6\%), lower limbs (48.6\%), and trunk (40.5\%). There were 4 cases with up to $90 \%$ of the TBSA and the third-degree burns. In total, 32 cases (86.5\%) underwent surgery. Eight (21.6\%) cases received more than 10 surgical procedures. The most common types of surgeries were operations on skin and subcutaneous tissues (66.2\%). The mean time spent of first surgery had $58.5 \pm 40.7 \mathrm{~min}$ and surgery during the 7 days before HAI had $76.3 \pm 57.3$ min (Table 1 ).

The mean age of the hospitalized burn cases was $22.0 \pm 6.2$ (range 13-38) years including 22 (59.5\%) males. Mean APACHE score was $14.3 \pm 8$ points; $10.8 \%$ had abnormal serum creatinine levels $(\geq 1.5 \mathrm{mg} / \mathrm{dL})$. There were $23(62.2 \%)$ cases with endotracheal tubes. Mean LOS before the first isolated MDRO $(n=23)$ was $17.7 \pm 16$ days. In total, 17 (45.9\%) cases developed HAI with 40 episodes. The most common type of HAI was bloodstream infections (BSIs; 70\%) with 28 episodes (Table 2). The mean Baux score was $24.5 \pm 8.7$ points with mortality rate of $2.7 \%$. One case $(70 \%$ TBSA and $49 \%$ third-degree burns) died with Baux score of 31 points, 4 months after admission. 
Table 1 Distribution of severity of burns and frequencies of surgery

\begin{tabular}{|c|c|c|}
\hline Variables & $\begin{array}{l}\text { Number } \\
(\boldsymbol{n}=37)\end{array}$ & $\begin{array}{l}\text { Percentage } \\
(\%)\end{array}$ \\
\hline $\begin{array}{l}\text { Number of total body surface area (\%) } \\
\text { (mean } \pm \text { SD; range) }\end{array}$ & $46.1 \pm 29.1$ & $5-92$ \\
\hline$<10$ & 6 & 16.2 \\
\hline $10-19$ & 4 & 10.8 \\
\hline $20-29$ & 2 & 5.4 \\
\hline $30-39$ & 1 & 2.7 \\
\hline $40-49$ & 5 & 13.5 \\
\hline $50-59$ & 6 & 16.2 \\
\hline $60-69$ & 3 & 8.1 \\
\hline $70-79$ & 4 & 10.8 \\
\hline $80-89$ & 2 & 5.4 \\
\hline$>90$ & 4 & 10.8 \\
\hline \multicolumn{3}{|l|}{ Number of third -degree burn severity (\%) } \\
\hline$<10$ & 3 & 8.1 \\
\hline $10-19$ & 1 & 2.7 \\
\hline $20-29$ & 3 & 8.1 \\
\hline $30-39$ & 1 & 2.7 \\
\hline $40-49$ & 3 & 8.1 \\
\hline $50-59$ & 4 & 10.8 \\
\hline $60-69$ & 4 & 10.8 \\
\hline $70-79$ & 3 & 8.1 \\
\hline $80-89$ & 1 & 2.7 \\
\hline$>90$ & 4 & 10.8 \\
\hline \multicolumn{3}{|l|}{ Main site of the third -degree burn } \\
\hline $\begin{array}{l}30-50 \% \text { burn of upper limb, except wrist } \\
\text { and hand }\end{array}$ & 18 & 48.6 \\
\hline $30-50 \%$ burn of lower limbs & 18 & 48.6 \\
\hline $30-50 \%$ burn of trunk & 15 & 40.5 \\
\hline 30-50\% burn of face, head, and neck & 10 & 27.0 \\
\hline $20-50 \%$ burn of wrists and hands & 4 & 10.8 \\
\hline $30-50 \%$ burn of multiple specified sites & 4 & 10.8 \\
\hline Unspecified degree & 10 & 27.0 \\
\hline \multicolumn{3}{|l|}{ Number of surgeries } \\
\hline 0 & 5 & 13.5 \\
\hline 1 & 7 & 18.9 \\
\hline 2 & 5 & 13.5 \\
\hline 3 & 7 & 18.9 \\
\hline 4 & 4 & 10.8 \\
\hline 5 & 3 & 8.1 \\
\hline 6 & 1 & 2.7 \\
\hline \multicolumn{3}{|l|}{ Number of various surgical procedures } \\
\hline 1 & 4 & 10.8 \\
\hline 2 & 1 & 2.7 \\
\hline
\end{tabular}

Table 1 Distribution of severity of burns and frequencies of surgery (Continued)

\begin{tabular}{lll}
\hline Variables & $\begin{array}{l}\text { Number } \\
(\boldsymbol{n}=37)\end{array}$ & $\begin{array}{l}\text { Percentage } \\
(\%)\end{array}$ \\
\hline 4 & 2 & 5.4 \\
5 & 2 & 5.4 \\
6 & 3 & 8.1 \\
7 & 4 & 10.8 \\
8 & 2 & 5.4 \\
9 & 1 & 2.7 \\
$>10$ & 8 & 21.6 \\
$\begin{array}{l}\text { Main types of surgery ( } n=142) \\
\quad \text { Operations on skin and subcutaneous }\end{array}$ & 94 & 66.2 \\
tissue & & \\
$\begin{array}{l}\text { Operations on muscle, tendon, and fascia } \\
\text { of hand }\end{array}$ & 18 & 12.7 \\
$\begin{array}{l}\text { Other operations on vessels } \\
\text { Time spent of first surgery (mean } \pm \text { SD; range) } \\
\text { (minutes) }\end{array}$ & $58.5 \pm 40.7$ & $5-215$ \\
$\begin{array}{l}\text { Time spent of surgery during the 7 days } \\
\text { before healthcare-associated infection } \\
\text { (mean } \pm \text { SD; range) (minutes) }\end{array}$ & $76.3 \pm 57.3$ & $8-269$ \\
\hline
\end{tabular}

\section{Microbiological investigations}

Table 3 lists the distribution of microbial isolates by TBSA and by days after admission. Out of 1132 clinical specimens, 706 were positive in cultures (62.4\%). A total of 335 strains of bacteria were isolated in the study. The three most commonly isolated groups of microorganisms were Gram-negative bacilli (GNB, $n=178$, 53.1\%) including glucose non-fermenting Gram-negative bacilli (GNFGNB, $n=132$, 39.4\%); Gram-positive bacteria ( $n=$ 101, 30.2\%) including Gram-positive cocci (GPC, $n=97$, $29 \%)$; and fungus $(n=51,15.2 \%)$. The most commonly isolated species include Staphylococcus spp. $\quad(n=75$, $22.4 \%)$, Acinetobacter spp. $(n=63,18.8 \%)$, and yeast $(n=45,13.4 \%)$. In addition, only $1.2 \%(n=4)$ of anaerobes were isolated. The MDRO accounted for $31 \%$ of all microbial isolates. The isolated MDROs began to increase in patients with TBSA $\geq 50 \%$ and hospitalization $\geq 15$ days. The most commonly isolated MDRO include carbapenem-resistant A. baumannii (CRAB, 14.6\% [imipenem-resistant $10.7 \%$ and pandrug-resistant $3.9 \%]$ ); MRSA (11.3\%); and carbapenem-resistant Klebsiella pneumoniae (CRKP, 2.4\%). In addition, pandrugresistant $P$. aeruginosa (PDRPA, 0.3\%) was isolated from one case of more than $70 \%$ TBSA and hospitalized for more than 31 days.

Figure 1 and Table 3 shows the trends in distribution of microbial isolates by TBSA. Overall, the total number of microbial isolates gradually increased with the increase in TBSA $(p<0.001)$. The OR peaked at 70-79\%TBSA (18.35), before declining to 12.45 at $80-89 \%$ TBSA. The decline in 
Table 2 Demographic and clinical characteristics of burn cases

\begin{tabular}{|c|c|c|}
\hline Variables & $\begin{array}{l}\text { Number }(\boldsymbol{n}= \\
37)\end{array}$ & $\begin{array}{l}\text { Percentage } \\
(\%)\end{array}$ \\
\hline Intensive care units & 24 & 64.9 \\
\hline \multicolumn{3}{|l|}{ Gender } \\
\hline Male & 22 & 59.5 \\
\hline Female & 15 & 40.5 \\
\hline Inhalation injury & 32 & 86.5 \\
\hline \multicolumn{3}{|l|}{ Laboratory data $^{a}$} \\
\hline White blood cell $\left(\geq 10,500 \mathrm{~mm}^{3}\right)$ & 27 & 73.0 \\
\hline Hematocrit $<35 \%$ & 8 & 21.6 \\
\hline Serum sodium ( $\geq 146 \mathrm{mmol} / \mathrm{L}$ ) & 6 & 16.2 \\
\hline Serum potassium ( $\geq 5.5 \mathrm{mmol} / \mathrm{L})$ & 4 & 10.8 \\
\hline Serum creatinine ( $\geq 1.5 \mathrm{mg} / \mathrm{dL})$ & 4 & 10.8 \\
\hline Serum albumin $(\leq 2.49 \mathrm{~g} / \mathrm{dL})$ & 0 & 0.0 \\
\hline \multicolumn{3}{|l|}{ Invasive devices (yes) } \\
\hline Endotracheal tube & 23 & 62.2 \\
\hline Mechanical ventilator $>96 \mathrm{~h}$ & 16 & 43.2 \\
\hline Central line catheter & 18 & 48.6 \\
\hline Foley catheter & 13 & 35.1 \\
\hline Hemodialysis & 2 & 5.4 \\
\hline $\begin{array}{l}\text { Extracorporeal membrane } \\
\text { oxygenation }\end{array}$ & 1 & 2.7 \\
\hline \multicolumn{3}{|c|}{ Site of healthcare-associated infection $(n=40)$} \\
\hline Bloodstream infection & 28 & 70.0 \\
\hline Symptomatic urinary tract infection & 9 & 22.5 \\
\hline Asymptomatic bacteriuria & 2 & 5.0 \\
\hline Central nervous system infection & 1 & 2.5 \\
\hline Mortality & 1 & 2.7 \\
\hline Continuity variables & mean $\pm S D$ & $\min -\max$ \\
\hline Age (years) & $22 \pm 6.2$ & $13-38$ \\
\hline APACHE II scores & $14.3 \pm 8.0$ & $2-32$ \\
\hline $\begin{array}{l}\text { LOS before the first isolated MDR }(n= \\
\text { 23) }\end{array}$ & $17.7 \pm 16.0$ & $2-55^{a}$ \\
\hline Total LOS in hospital stay ${ }^{\#}$ & $85.4 \pm 70.4$ & $9-276^{\mathrm{a}}$ \\
\hline Baux score & $24.5 \pm 8.7$ & $6-46$ \\
\hline
\end{tabular}

SD standard deviation, APACHE acute physiology and chronic health evaluation, $L O S$ length of stay, MDR multi-drug resistant microbes

${ }^{a}$ The highly abnormal values during the hospital stay were selected for the analysis

"The LOS is calculated throughout the hospital stay, and other data are within 3 months of hospitalization (from June 27 to October 31)

OR slowed to 10.54 at more than $90 \%$ TBSA. At $39 \%$ or less TBSA, significant change was observed for GPC $(p=$ 0.027), however the OR decreased with increasing TBSA and maintained between 0.17-0.31. Gram-positive bacilli isolates (only 4 strains) first appeared at $50-59 \%$ TBSA, the OR also decreased with increasing TBSA $(p=0.014)$.

Figure 2 indicate the trends in microbial isolation by days after admission. Overall, the total number of microbial isolates gradually increased with the increase in the hospitalization days $(p<0.001)$. The OR $(4.50)$ of microbial isolates increased markedly in 15-30 hospitalization days and the OR (9.80) reached peak at 31 hospitalization days or more. Gram-positive bacilli showed statistically significant change $(p<0.001)$, which was the highest in 1-3 hospitalization days, but the OR of isolates decreased with increasing hospitalization days. For all other microbial isolates, such as GPC, GNB, GNFGNB, anaerobes and fungi, no statistically significant difference $(p>0.05)$ in OR trends was observed. The OR (6.82) of the fungal isolates reached the highest at 4-7 hospitalization days, followed by a slight decrease but remained stable (3.05-5.69) thereafter.

\section{Main risk factors}

Logistic and Cox regression were used to analyze potential risk factors for MDRO (Table 4). In the simple logistic and Cox models, the TBSA showed statistically significant difference $(p=0.003)$, while the third-degree burn severity showed statistically significant difference $(p=0.002)$ in the logistic model, but no statistical difference was observed in Cox Model $(p=0.076)$. In the multiple regressions, for each additional $10 \%$ of the TBSA, the MDRO increased 17.57 times (95\% CI [1.47-21.0], $p=0.024$ ) by logistic model and 2.58 times $(95 \% \mathrm{CI}$ [1.71-3.88], $p<0.001)$ by Cox model. For each additional $10 \%$ increase in third-degree burn severity, the MDRO decreased significantly by $47 \%$ (95\% CI [0.380.73 ] $p<0.001)$ by Cox model. In addition, surgical intervention was not statistically significant in isolating $\operatorname{MDRO}(p=0.057)$.

\section{Discussion}

This study was conducted on young population that suffered burns due to single dust explosion event. Given high homogeneity among cases, our results demonstrated that the TBSA range and the hospitalization days were independent factors for the isolation of MDRO. However, the severity of burns and the time elapsed after burns influenced the various microbial isolates differently.

\section{Microbes and drug-resistant bacteria}

Several reports indicated that the most common genera isolated from wound or burn units were GPC, followed by GNB $[1,2,6,7]$. However, the results from this study differed from previously published reports. We found the most commonly isolated group was GNB (53.1\%). Among GNB, the most commonly isolated subgroup was GNFGNB (39.4\%). This difference could be attributed to differences in the type of burns, populations, age distribution, and data analysis period. However, the most common bacteria isolated in this study were Acinetobacter 
Table 3 Distribution of microbial isolates by total body surface area and by days after admission

\begin{tabular}{|c|c|c|c|c|c|c|c|c|c|c|c|c|c|c|}
\hline \multirow[t]{2}{*}{ Isolated pathogens } & \multirow[t]{2}{*}{ Total } & \multirow[t]{2}{*}{$\%$} & \multicolumn{7}{|c|}{ Total body surface area (\%) } & \multicolumn{5}{|c|}{ days after admission } \\
\hline & & & $<39$ & $40-49$ & $50-59$ & $60-69$ & $70-79$ & $80-89$ & $>90$ & $1-3$ & $4-7$ & $8-14$ & $15-30$ & $>31$ \\
\hline Total sampling numbers of culture specimen & 706 & & 28 & 65 & 118 & 108 & 151 & 142 & 94 & 66 & 38 & 78 & 162 & 362 \\
\hline Total number of culture-positive sample & 335 & 100 & 6 & 29 & 43 & 58 & 84 & 62 & 54 & 26 & 28 & 38 & 92 & 151 \\
\hline Rate of culture-positive (\%) & 47.5 & & 21.4 & 44.6 & 36.4 & 53.7 & 55.6 & 43.7 & 57.4 & 39.4 & 73.7 & 48.7 & 56.8 & 41.7 \\
\hline Total number of MDRO & 104 & & 1 & 9 & 17 & 21 & 20 & 19 & 17 & 2 & 6 & 9 & 35 & 52 \\
\hline Rate of MDRO in total culture-positive (\%) & 31.0 & & 0.96 & 8.7 & 16.3 & 20.2 & 19.2 & 18.3 & 16.3 & 1.9 & 5.8 & 8.7 & 33.7 & 50.0 \\
\hline Gram-positive cocci & 97 & 29.0 & 4 & 11 & 16 & 18 & 21 & 11 & 17 & 7 & 3 & 12 & 26 & 49 \\
\hline Staphylococcus sp. & 75 & 22.4 & 3 & 10 & 12 & 15 & 13 & 8 & 14 & 2 & 3 & 11 & 23 & 36 \\
\hline S. aureus & 2 & 0.6 & 1 & 1 & 0 & 0 & 0 & 0 & 0 & 0 & 0 & 1 & 0 & 1 \\
\hline S. aureus (MRSA) & 38 & 11.3 & 1 & 4 & 8 & 9 & 4 & 6 & 6 & 0 & 2 & 1 & 11 & 24 \\
\hline coagulase-negative staphylococcus & 21 & 6.3 & 1 & 2 & 3 & 4 & 6 & 1 & 4 & 2 & 1 & 7 & 6 & 5 \\
\hline other Staphylococcus sp. & 14 & 4.2 & 0 & 3 & 1 & 2 & 3 & 1 & 4 & 0 & 0 & 2 & 6 & 6 \\
\hline Enterococcus sp. & 9 & 2.7 & 1 & 1 & 2 & 3 & 2 & 0 & 1 & 4 & 0 & 1 & 0 & 4 \\
\hline E. faecium (VRE) & 7 & 2.1 & 0 & 0 & 1 & 0 & 4 & 2 & 0 & 1 & 0 & 0 & 2 & 4 \\
\hline Others & 6 & 1.8 & 0 & 0 & 1 & 0 & 2 & 1 & 2 & 0 & 0 & 0 & 1 & 5 \\
\hline Gram-positive bacilli & 4 & 1.2 & 0 & 0 & 3 & 0 & 1 & 0 & 0 & 2 & 2 & 0 & 0 & 0 \\
\hline Gram-negative bacilli & 46 & 13.7 & 1 & 3 & 7 & 7 & 11 & 8 & 9 & 4 & 3 & 7 & 12 & 20 \\
\hline \multicolumn{15}{|l|}{ Enterobacteriaceae } \\
\hline Klebsiella pneumoniae & 9 & 2.7 & 0 & 0 & 3 & 2 & 1 & 2 & 1 & 0 & 1 & 1 & 3 & 4 \\
\hline Klebsiella pneumonia (CRKP) & 8 & 2.4 & 0 & 0 & 0 & 4 & 1 & 1 & 2 & 0 & 0 & 1 & 2 & 5 \\
\hline Escherichia coli & 5 & 1.5 & 0 & 1 & 0 & 0 & 1 & 1 & 2 & 0 & 0 & 0 & 3 & 2 \\
\hline Enterobacter sp. & 4 & 1.2 & 1 & 1 & 0 & 0 & 2 & 0 & 0 & 1 & 1 & 0 & 1 & 1 \\
\hline E. cloacae (CRE) & 1 & 0.3 & 0 & 0 & 1 & 0 & 0 & 0 & 0 & 1 & 0 & 0 & 0 & 0 \\
\hline Proteus mirabilis & 4 & 1.2 & 0 & 0 & 1 & 0 & 2 & 0 & 1 & 0 & 0 & 0 & 2 & 2 \\
\hline Serratia marcescens & 3 & 0.9 & 0 & 0 & 1 & 0 & 1 & 0 & 1 & 2 & 0 & 0 & 1 & 0 \\
\hline Aeromonas hydrophila & 2 & 0.6 & 0 & 1 & 0 & 0 & 1 & 0 & 0 & 0 & 1 & 1 & 0 & 0 \\
\hline Others & 10 & 3.0 & 0 & 0 & 1 & 1 & 2 & 4 & 2 & 0 & 0 & 4 & 0 & 6 \\
\hline Non-fermentative Gram-negative bacilli & 132 & 39.4 & 0 & 10 & 17 & 22 & 32 & 27 & 24 & 10 & 14 & 13 & 44 & 51 \\
\hline Acinetobacter sp. & 63 & 18.8 & 0 & 8 & 9 & 12 & 13 & 12 & 9 & 3 & 6 & 9 & 26 & 19 \\
\hline A. baumannii & 1 & 0.3 & 0 & 0 & 0 & 1 & 0 & 0 & 0 & 0 & 1 & 0 & 0 & 0 \\
\hline A. baumannii (IRAB) & 36 & 10.7 & 0 & 5 & 5 & 8 & 6 & 6 & 6 & 0 & 3 & 7 & 18 & 8 \\
\hline A. baumannii (PDRAB) & 13 & 3.9 & 0 & 0 & 2 & 0 & 4 & 4 & 3 & 0 & 1 & 0 & 2 & 10 \\
\hline A. baumannii complex & 13 & 3.9 & 0 & 3 & 2 & 3 & 3 & 2 & 0 & 3 & 1 & 2 & 6 & 1 \\
\hline Pseudomonas aeruginosa & 33 & 9.9 & 0 & 0 & 3 & 5 & 10 & 6 & 9 & 2 & 3 & 0 & 10 & 18 \\
\hline P. aeruginosa (PDR-PA) & 1 & 0.3 & 0 & 0 & 0 & 0 & 1 & 0 & 0 & 0 & 0 & 0 & 0 & 1 \\
\hline Stenotrophomonas maltophilia & 13 & 3.9 & 0 & 1 & 2 & 1 & 3 & 3 & 3 & 2 & 2 & 1 & 4 & 4 \\
\hline Chryseobacterium indologenes & 7 & 2.1 & 0 & 1 & 0 & 1 & 2 & 2 & 1 & 2 & 0 & 1 & 0 & 4 \\
\hline C. memingosepticum & 5 & 1.5 & 0 & 0 & 0 & 1 & 2 & 1 & 1 & 1 & 2 & 1 & 1 & 0 \\
\hline Chryseobacterium spp. & 4 & 1.2 & 0 & 0 & 0 & 1 & 1 & 2 & 0 & 0 & 0 & 0 & 0 & 4 \\
\hline Burkholderia cepacia & 3 & 0.9 & 0 & 0 & 3 & 0 & 0 & 0 & 0 & 0 & 0 & 1 & 2 & 0 \\
\hline Pseudomonas putida & 1 & 0.3 & 0 & 0 & 0 & 1 & 0 & 0 & 0 & 0 & 1 & 0 & 0 & 0 \\
\hline Moraxella osloensis & 1 & 0.3 & 0 & 0 & 0 & 0 & 0 & 1 & 0 & 0 & 0 & 0 & 1 & 0 \\
\hline Moraxella morganii & 1 & 0.3 & 0 & 0 & 0 & 0 & 0 & 0 & 1 & 0 & 0 & 0 & 0 & 1 \\
\hline Anaerobes & 4 & 1.2 & 0 & 1 & 0 & 0 & 0 & 2 & 0 & 2 & 0 & 0 & 0 & 2 \\
\hline
\end{tabular}


Table 3 Distribution of microbial isolates by total body surface area and by days after admission (Continued)

\begin{tabular}{|c|c|c|c|c|c|c|c|c|c|c|c|c|c|c|}
\hline \multirow[t]{2}{*}{ Isolated pathogens } & \multirow[t]{2}{*}{ Total } & \multirow[t]{2}{*}{$\%$} & \multicolumn{7}{|c|}{ Total body surface area (\%) } & \multicolumn{5}{|c|}{ days after admission } \\
\hline & & & $<39$ & $40-49$ & $50-59$ & $60-69$ & $70-79$ & $80-89$ & $>90$ & $1-3$ & $4-7$ & $8-14$ & $15-30$ & $>31$ \\
\hline Fungus & 51 & 15.2 & 1 & 4 & 0 & 11 & 19 & 13 & 4 & 1 & 6 & 6 & 10 & 28 \\
\hline Yeast & 45 & 13.4 & 0 & 3 & 0 & 10 & 15 & 13 & 4 & 1 & 6 & 4 & 8 & 26 \\
\hline Yeast & 25 & 7.5 & 0 & 1 & 0 & 8 & 7 & 9 & 0 & 0 & 2 & 2 & 4 & 17 \\
\hline Candida spp. & 14 & 4.2 & 0 & 2 & 0 & 2 & 5 & 2 & 3 & 1 & 3 & 2 & 4 & 4 \\
\hline Others & 6 & 1.8 & 0 & 0 & 0 & 0 & 3 & 2 & 1 & 0 & 1 & 0 & 0 & 5 \\
\hline MOLD & 6 & 1.8 & 0 & 1 & 0 & 1 & 4 & 0 & 0 & 0 & 0 & 2 & 2 & 2 \\
\hline
\end{tabular}

MDRO multiple drug-resistant organisms, MRSA methicillin-resistant $S$. aureus, VRE vancomycin-resistant Enterococcus, CRKP carbapenem-resistant $K$. pneumonia, $C R E$, carbapenem-resistant Enterobacteriaceae, IRAB imipenem-resistant $A$. baumannii, PDRAB pandrug-resistant $A$. baumannii, $P D R-P A$ pandrug-resistant P. aeruginosa

*PDRAB was defined as being resistant to aminoglycosides, antipseudomonal carbapenems, antipseudomonal fluoroquinolones, antipseudomonal penicillins+ $\beta$ lactamase inhibitors, extended-spectrum cephalosporins, folate pathway inhibitors, penicillins+ $\beta$-lactamase inhibitors, polymyxins, tetracycline

*PDRPA was defined as being resistant to aminoglycosides, antipseudomonal carbapenems, antipseudomonal cephalosporins, antipseudomonal fluoroquinolones, antipseudomonal penicillins+ $\beta$-lactamase inhibitors, monobactams, phosphonic acids, polymyxins

spp. and $P$. aeruginosa, which is consistent with a majority of other published reports $[1,2,7,9]$. Interestingly, those two bacteria along with Stenotrophomonas maltophilia were isolated within 3 days of hospitalization. One study on similar population affected by dust explosion but where patients were transmitted to other hospitals for treatment, found that Ralstonia pickettii (18.5\%) was one of the most common bacteria causing BSIs [20]. These bacteria survive easily in sewage or humid environments.

Among MDRO, Gram-positive bacteria particularly MRSA continues to be an important pathogen in burn infections $[1,4]$. Our data showed lower frequency of isolation by S. aureus (11.3\%) compared to MRSA (95\%). MRSA cases showed TBSA of more than $40 \%$ and were hospitalized for more than 15 days. This result was in contradiction with another study that monitored all hospitalized burn patients for 1 year and found only $16.7 \%$ $S$. aureus strains resistant to methicillin. However, burn patients in that study merely had a median TBSA of $7 \%$ (interquartile range $4-14 \%$ ) and a median hospital stay of 14 days [7]. Apart from MRSA, care and treatment of burn patients infected with VRE has also become increasingly challenging. Isolation rate of VRE in the present study was low $(2.1 \%)$. The reason could be younger healthy population enrolled in the present study coupled with proper antibiotic management and infection control.

Gram-negative bacteria have increasingly become highly resistant to antimicrobial agents due to their ability to form biofilms that appear to enhance their

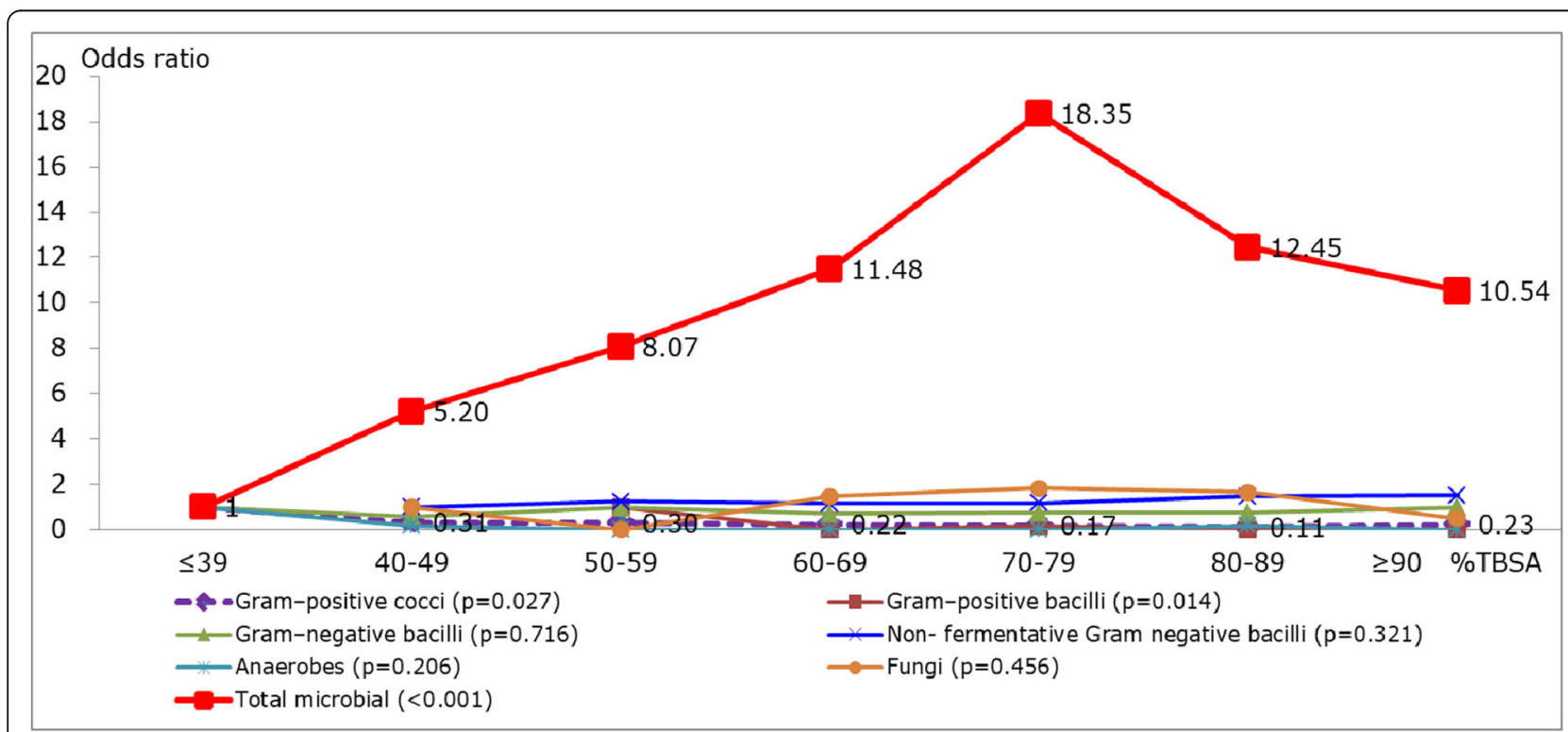

Fig. 1 Trends in distribution of microbial isolates by total body surface area in the culture-positive specimens 


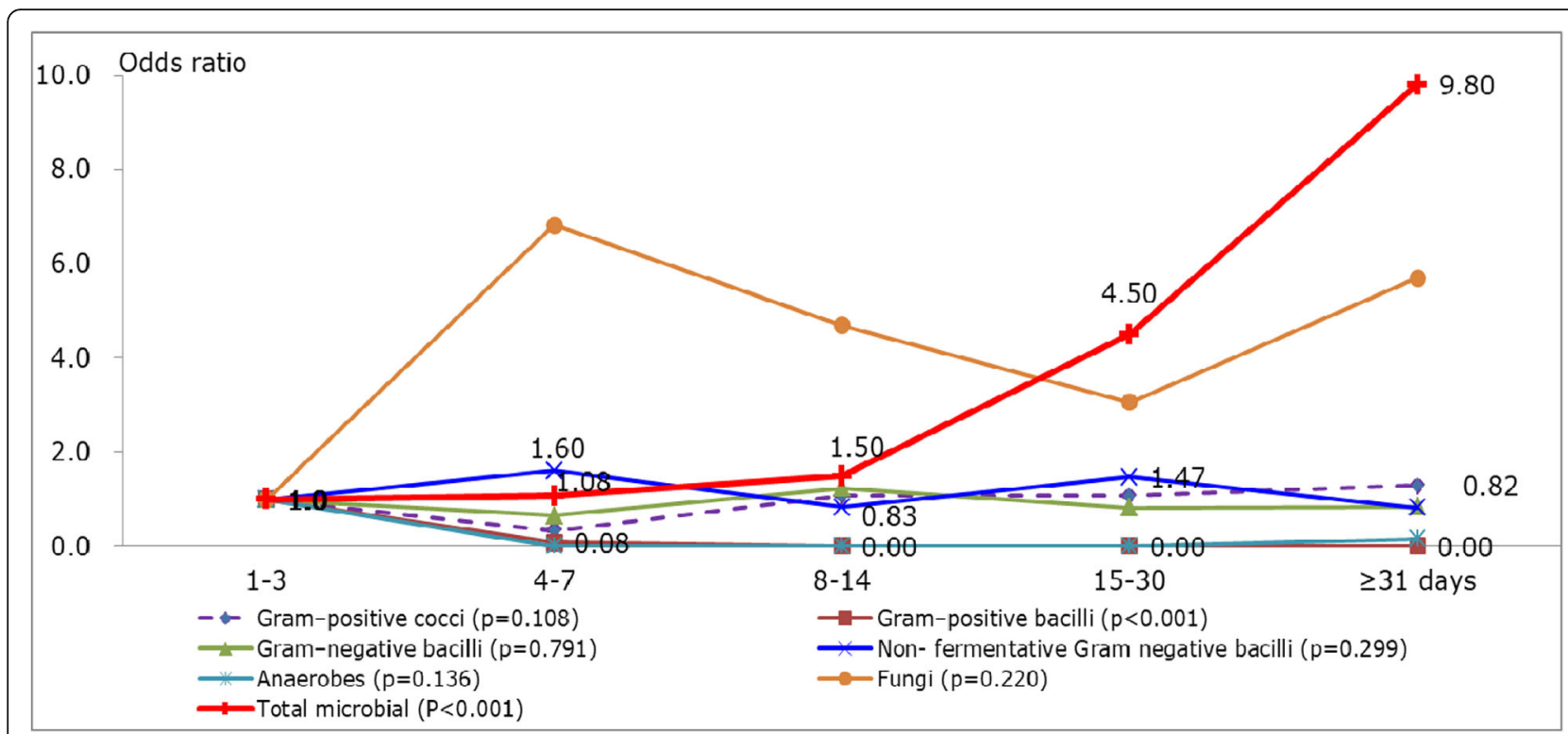

Fig. 2 Trends in the main microbial isolates by days after admission in the hospital (Culture positive specimens)

pathogenicity [21]. Shoja et al. found a high rate (92.5\%) of isolation of CRAB [22]. However, their report lacked important parameters such as the range and depth of burns, and the distribution of age. Our data displayed that in all types of ICU, the IRAB BSIs of burn $\mathrm{CU}$ increased during the first month after hospitalization. The TBSA in those cases ranged from 67 to $>90 \%$, and those infections occurred 6-17 days after admission. More information might be provided if molecular epidemiological surveillance was available. However, the overall data from our study showed that the incidence of $A$. baumannii isolates resistant to carbapenem was relatively low, and the result was similar to the incidence of CRABs (21\%) reported by two similar groups, but treated in different hospitals $[23,24]$. Apart from CRAB, the resistance rates for other GNB were also quite low, such as carbapenem-resistant Enterobacteriaceae (0.3-2.4\%) and pandrug-resistant $P$. aeruginosa (0.3\%). Antimicrobial stewardship is extremely important in burn units [25]. In order to reduce microorganisms and MDRO in the environment, our study not only formulated cleaning procedures and educating cleaners, also used auditing procedures such as use of luminometers to ensure proper disinfection and cleanliness to reduce the risk of microbial and MDRO presence and transmission.

\section{Main risk factors for microbial colonization and infection}

In the present study, besides the endogenous and exogenous microbial colonization or infection there were other risk factors that could promote microbial infection and increased resistance to antibiotics in burn patients. Due to the fact that subjects in this study belonged to a young and homogeneous group, of particular interest to us was the influence of both burn severity and time factor on the types of microbial isolates. Several multivariate analyses of prospective or retrospective studies have revealed that the degree of burn wounds was a major risk factor for microbial colonization and infection $[1,5,7,26]$. Compared to $\leq 19 \%$ of TBSA, patients with greater than $20 \%$ of TBSA were more than twice (RR 2.09-2.41) likely to be infected at a rate that was five times faster [7]. Several studies revealed that patients with TBSA range greater than $25 \%$ or greater than $30 \%$ had a high risk of microbial infection, because these patients usually need to use invasive devices for treatment $[4,26]$. One report suggested that infection rates rose by an increase in TBSA of burn; they observed infection rates of 18,30 and $52 \%$ in

Table 4 Regression models of risk factors for multi-drug resistant microbes

\begin{tabular}{|c|c|c|c|c|c|c|c|c|}
\hline \multirow[t]{3}{*}{ Variables } & \multicolumn{4}{|c|}{ Logistic regression model } & \multicolumn{4}{|c|}{ Cox regression model } \\
\hline & \multicolumn{2}{|l|}{ Unadjust } & \multicolumn{2}{|l|}{ Adjust } & \multicolumn{2}{|l|}{ Unadjust } & \multicolumn{2}{|l|}{ Adjust } \\
\hline & $\begin{array}{l}\mathrm{OR} \\
(95 \% \mathrm{Cl})\end{array}$ & $p$-value & $\begin{array}{l}\mathrm{OR} \\
(95 \% \mathrm{Cl})\end{array}$ & $p$-value & $\begin{array}{l}\mathrm{HR} \\
(95 \% \mathrm{Cl})\end{array}$ & $p$-value & $\begin{array}{l}\mathrm{HR} \\
(95 \% \mathrm{Cl})\end{array}$ & $p$-value \\
\hline Total body surface area (every 10\%) & $3.6(1.6-8.2)$ & .003 & $17.57(1.47-21.0)$ & .024 & $1.31(1.01-1.56)$ & .003 & $2.58(1.71-3.88)$ & $<.001$ \\
\hline Third burn-degree severity (every 10\%) & $1.79(1.2-2.6)$ & .002 & $0.26(0.05-1.41)$ & .118 & $1.14(0.99-1.32)$ & .076 & $0.53(0.38-0.73)$ & $<.001$ \\
\hline
\end{tabular}


patients with $15-30 \%, 31-50 \%$ and $51-69 \%$ of TBSA respectively [5]. In the present study, patients with severe burns cases had the average TBSA of $46.1 \%$; however, infection rates for patients with greater than $50 \%$ TBSA rose by $56.8 \%$.

Unexpectedly, our data showed the number of isolated GPC were the highest in patients with less than 39\% TBSA, and the proportion of isolated GPC showed a downward trend $(p=0.027)$ with the increase in TBSA extent. However, in terms of overall isolated microorganisms, our data were consistent with previously published reports $[1,5,7]$. We found an upward trend $(p<$ $0.001)$ in the proportion of overall microbial isolates over time, until the TBSA reached $79 \%$. Afterwards, the trend stabilized followed by a decline. In general, the risk of death of patients with severe burns was relatively high and rapid, so overall isolated microbial maybe thereby reduced. However, our data could not confirm this statement due to death in only one case (70\% TBSA). In terms of the overall MDRO, we also used regression analyses approach to adjust the depth of burns, the likelihood of infections by MDRO increased by $2.58-17.57$ times for each $10 \%$ increase in TBSA.

Another important variable related to severity of burn is the burn depth. One report indicated that the thirddegree burns would significantly influence (HR 7.88) the burn wound infection. They concluded that risk of infection for patients with third-degree burns almost doubled and patients got infected at a faster rate [7]. Another study using multiple analysis revealed that only the third-degree burn (OR 1.10, 95\% CI, 1.02-1.19, $p=$ 0.012) was an independent risk factor [23]. Using univariable analysis, our results demonstrated significantly higher likelihood of MDRO infections (1.14-1.79 times) for every $10 \%$ increase in the third-degree burn severity. However, when the multiple analyses were used after adjusted TBSA variable, the risk of MDRO was reduced by $47-74 \%$. This may be linked to early excision, debridement and grafting, use special materials dressings to treatment, as well as appropriate antibiotic use and infection control, which could reduce the risk of MDRO and cross-contamination in patients with deep burns.

The time factor is also one of the important factors influencing colonization or infection of predominant microorganisms in burn patients [2]. Generally, early colonization of the wound in the first $48 \mathrm{~h}$ occur with Gram-positive bacteria from the endogenous skin flora [1]. Next, Gram-positive bacteria are replaced by Gramnegative bacteria by the second week [2]. Hereafter, if the wound closure is delayed and the patient becomes infected and is treated with broad-spectrum antibiotics, a further shift in flora towards yeasts, fungi, and antibiotic-resistant bacteria takes place [4].
Devrim et al. found that the median duration of development of BSIs caused by GPBs from the time of burn was 5 days (ranging from 2 to 54 days after burn), significantly less than that of BSIs caused by GNB (12 days) and fungal pathogens (13 days) [6]. Recently one study showed that the overwhelming majority of bloodstream pathogens were GNB isolates typically isolated within 2 weeks after the burn. During 15-28 days after burn, A. baumannii was the most common causative pathogen, while Chryseobacterium spp., S. maltophilia, and R. mannitolilytica were most frequently isolated early (mean: 14.5 days) after the burn [24]. The proportion of the overall microbial isolates in this study showed a significant change in trend over time; in particular, hospitalization for more than 14 days (OR 4.59.8, $p<0.001)$. Our findings correlated well with previous studies with reference to isolation of GPBs; nearly a half of them (only 4 strains) were isolated within 3 days of hospitalization, before showing downward trend $(p<0.001)$. Although there was no statistically significant difference in the trend for the proportion of GNFGNB isolation $(p=$ 0.299), with Acinetobacter spp. (71.4\%) and P. aeruginosa $(84.8 \%)$ being two major groups isolated at 15 or more days after hospitalization.

One of the major strengths of this study was that it adopted stratification analysis to examine trends in major microbial isolates based on the severity of the burn and time elapsed after burn. However, our study also had some limitations. First, the sample size of cases with severe burns was relatively small, although the source of the samples was single dust explosion event. The cases had homogeneity including patient age, medical treatment and care, and environmental setting factors. Therefore, our results successfully demonstrated the trends in microbial isolation and important factors that govern the rate of isolation. Second, the dispersion of the patients in different 5 ICUs and burn ward might cause some heterogeneity of the sampling. However, an interdisciplinary medical team was quickly established and held regular meetings on treatment and disposal strategies to achieve consensus and consistency. Third, this manuscript was mainly based on recommended of the US CDC's definition of burn infection, including changes in the appearance or characteristics of burn wound. Burn injury could result in severe sepsis and septic shock; however, our database could not display the data.

Finally, biomarkers can be used to monitor various aspects of disease progression and patient health. The World Health Organization proposed a broad definition of biomarker including almost any measurement reflecting an interaction between a biological system and a potential hazard, which may be chemical, physical, or biological. Thus, the biomarkers as an indicator can be objectively measured and evaluated, such as single 
molecules, protein panels, injury characteristics, or clinical parameters that may affect clinical outcomes in the severely burned [27]. We suggest that future research can be explored in other aspects, including the impact of surgical intervention and antimicrobial stewardship on the type of microorganisms isolated, and perform molecular typing of microbial isolates.

\section{Conclusion}

We found that the proportion of overall microbial isolates increased with increase in the extent of TBSA and the time elapsed after burns. The extent of TBSA was the most important factor affecting MDRO. The study demonstrated that under well-integrated and operational medical and infection control teams, microbial transmission could be reduced to improve the survival rates of burn patients.

\section{Abbreviations}

BSI: Bloodstream infection; Cl: Confidence interval; CRAB: Carbapenemresistant Acinetobacter baumannii; CRKP: Carbapenem-resistant Klebsiella pneumoniae; ESBL: Extended-spectrum beta-lactamase; GNB: Gram-negative bacillus; GNFGNB: Glucose non-fermenting Gram-negative bacillus; GPC: Gram-positive coccus; HAl: Healthcare-associated infection; HR: Hazard ratio; ICU: Intensive care unit; MDRO: Multiple drug-resistant organism; MRSA: Methicillin-resistant Staphylococcus aureus; OR: Odds ratio; TBSA: Total body surface area; VRE: Vancomycin-resistant Enterococcus

\section{Acknowledgements}

We pay tribute to the frontline medical team of the Taipei Veterans Genera Hospital, Taiwan.

\section{Authors' contributions}

YYC and FDW participated in the design, led the analysis, and drafted the manuscript. PFW participated in substantively revising the manuscript. CSC and $\mathrm{IHC}$ participated in the analysis and interpretation and drafted the Tables and Figures of the manuscript. WTH participated in the data collection. FDW commented and revised on draft of the manuscript. All authors approved the final manuscript.

\section{Funding}

This work was supported by a research grant from Taipei Veterans General Hospital, Taipei, Taiwan (grant number V107C-208 and V107C-169). The funding institutes did not have any role in study design, data collection/analysis, the writing of the manuscript or the decision to submit the manuscript for publication.

\section{Availability of data and materials}

The datasets used and/or analyzed during the current study are available from the corresponding author on reasonable request.

\section{Ethics approval and consent to participate}

This study was approved by the Institution Review Board of Taipei Veterans General Hospital. The informed consent was waived in this study; however, the data used was anonymised before its use.

\section{Consent for publication}

Not applicable.

\section{Competing interests}

The authors declare that they have no competing interests.

\section{Author details}

'Department of Infection Control, Taipei Veterans General Hospital, Taipei, Taiwan. ${ }^{2}$ Department of Nursing, Taipei Veterans General Hospital, Taipei, Taiwan. ${ }^{3}$ College of Nursing, National Yang-Ming University, Taipei, Taiwan.
${ }^{4}$ Division of Infectious Diseases, Department of Medicine, Taipei Veterans General Hospital, Taipei, Taiwan. ${ }^{5}$ School of Medicine, National Yang-Ming University, Taipei, Taiwan. ${ }^{6}$ Taipei City Hospital, Taipei, Taiwan. ${ }^{7}$ Institute of Public Health, and Community Medicine Research Center, National Yang-Ming University, Taipei, Taiwan.

Received: 15 April 2019 Accepted: 26 February 2020

Published online: 04 March 2020

\section{References}

1. Mayhall CG. Healthcare-associated burn wound infections. In: Mayhall CG, editor. Hospital epidemiology and infection control. Philadelphia: Lippincott Willams \& Wilkins; 2012. p. 338-51.

2. Mozingo DW, Pruitt BA. Infections of burn wounds. In: Jarvis WR, editor. Bennett \& Brachman's hospital infections. Philadelphia: Lippincott Willams \& Wilkins; 2014. p. 552-60.

3. Siegel JD, Rhinehart E, Jackson M, Chiarello L, Healthcare Infection Control Practices Advisory C. Management of multidrug-resistant organisms in health care settings, 2006. Am J Infect Control. 2007;35:S165-93.

4. Weber J, McManus A, Nursing Committee of the International Society for Burn I. Infection control in burn patients. Burns. 2004;30:A16-24.

5. Ronat JB, Kakol J, Khoury MN, Berthelot M, Yun O, Brown V, et al. Highly drug-resistant pathogens implicated in burn-associated bacteremia in an Iraqi burn care unit. PLoS One. 2014;9:e101017.

6. Devrim I, Kara A, Duzgol M, Karkiner A, Bayram N, Temir G, et al. Burnassociated bloodstream infections in pediatric burn patients: time distribution of etiologic agents. Burns. 2017;43:144-8.

7. Ramirez-Blanco CE, Ramirez-Rivero CE, Diaz-Martinez LA, Sosa-Avila LM. Infection in burn patients in a referral center in Colombia. Burns. 2017;43: $642-53$.

8. Merchant N, Smith K, Jeschke MG. An ounce of prevention saves tons of lives: infection in burns. Surg Infect. 2015;16:380-7.

9. Siegel JD, Rhinehart E, Jackson M, Chiarello L, Health Care Infection Control Practices Advisory C. 2007 guideline for isolation precautions: preventing transmission of infectious agents in health care settings. Am J Infect Control. 2007:35:565-164.

10. Lachiewicz AM, van Duin D, DiBiase LM, Jones SW, Carson S, Rutala WA et al. Rates of hospital-associated respiratory infections and associated pathogens in a regional burn center, 2008-2012. Infect Control Hosp Epidemiol. 2015;36:601-3.

11. Chen YY, Chou YC, Chou P. Impact of nosocomial infection on cost of illness and length of stay in intensive care units. Infect Control Hosp Epidemiol. 2005;26:281-7.

12. Chen YY, Wang FD, Liu CY, Chou P. Incidence rate and variable cost of nosocomial infections in different types of intensive care units. Infect Control Hosp Epidemiol. 2009;30:39-46.

13. Hop MJ, Polinder S, van der Vlies CH, Middelkoop E, van Baar ME. Costs of burn care: a systematic review. Wound Repair Regen. 2014;22:436-50.

14. Stylianou N, Buchan I, Dunn KW. A review of the international Burn Injury Database (iBID) for England and Wales: descriptive analysis of burn injuries 2003-2011. BMJ Open. 2015;5:e006184.

15. Trop M, Herzog SA, Pfurtscheller K, Hoebenreich AM, Schintler MV, Stockenhuber A, et al. The past 25 years of pediatric burn treatment in Graz and important lessons been learned. An overview. Burns. 2015;41:714-20.

16. Smolle C, Cambiaso-Daniel J, Forbes AA, Wurzer P, Hundeshagen G, Branski $L K$, et al. Recent trends in burn epidemiology worldwide: a systematic review. Burns. 2017:43:249-57.

17. Oncul O, Oksuz S, Acar A, Ulkur E, Turhan V, Uygur F, et al. Nosocomial infection characteristics in a burn intensive care unit: analysis of an elevenyear active surveillance. Burns. 2014;40:835-41.

18. Ministry of Health and Welfare (MOHW). Details of the first meeting of the MOHW task force in response to the Formosa Fun Coast Dust Explosion. Taiwan: MOHW; 2017. https://www.mohw.gov.tw/cp-117-386-2.html. Accessed 1 July 2018.

19. Dellinger RP, Levy MM, Rhodes A, Annane D, Gerlach H, Opal SM, et al. Surviving sepsis campaign: international guidelines for management of severe sepsis and septic shock: 2012. Crit Care Med. 2013;41:580-637.

20. Horan TC, Andrus M, Dudeck MA. CDC/NHSN surveillance definition of health care-associated infection and criteria for specific types of infections in the acute care setting. Am J Infect Control. 2008;36:309-32. 
21. Trostrup H, Lerche CJ, Christophersen L, Thomsen K, Jensen PO, Hoiby N, et al. Pseudomonas aeruginosa biofilm infection suppresses local host response in burn wounds. Wound Repair Regen. 2015;23:A31.

22. Shoja S, Moosavian M, Rostami S, Farahani A, Peymani A, Ahmadi K, et al. Dissemination of carbapenem-resistant Acinetobacter baumannii in patients with burn injuries. J Chin Med Assoc. 2017;80:245-52

23. Lin TC, Wu RX, Chiu CC, Yang YS, Lee Y, Lin JC, et al. The clinical and microbiological characteristics of infections in burn patients from the Formosa fun coast dust explosion. J Microbiol Immunol Infect. 2018;51:26777.

24. Huang PY, Shie SS, Ye JJ, Lin SP, Liu TP, Wu TS, et al. Acquisition and clearance of multidrug resistant Acinetobacter baumannii on healthy young adults concurrently burned in a dust explosion in Taiwan: the implication for antimicrobial stewardship. BMC Infect Dis. 2017;17:598.

25. Lin YT. What can we learn from the dissemination of carbapenem-resistant Acinetobacter baumannii in patients with burn injury? J Chin Med Assoc. 2017:80:189-90.

26. Tekin R, Dal T, Bozkurt F, Deveci O, Palanc Y, Arslan E, et al. Risk factors for nosocomial burn wound infection caused by multidrug resistant Acinetobacter baumannii. J Burn Care Res. 2014;35:e73-80.

27. Ruiz-Castilla M, Roca O, Masclans JR, Barret JP. Recent advances in biomarkers in severe burns. Shock. 2016:45:117-25.

\section{Publisher's Note}

Springer Nature remains neutral with regard to jurisdictional claims in published maps and institutional affiliations.

Ready to submit your research? Choose BMC and benefit from:

- fast, convenient online submission

- thorough peer review by experienced researchers in your field

- rapid publication on acceptance

- support for research data, including large and complex data types

- gold Open Access which fosters wider collaboration and increased citations

- maximum visibility for your research: over $100 \mathrm{M}$ website views per year

At BMC, research is always in progress.

Learn more biomedcentral.com/submissions 